\title{
Optimal Resource Allocation Scheme in a Multirate Overlapped Optical CDMA System
}

\author{
Robert Raad, Elie Inaty, Member, IEEE, Paul Fortier, Senior Member, IEEE, and \\ Hossam M. H. Shalaby, Senior Member, IEEE
}

\begin{abstract}
This paper addresses the problem of resource allocation in a multiservice optical network based on an overlapped code-division-multiple-access system. A joint transmission power and overlapping coefficient (transmission rate) allocation strategy is provided via the solution of a constrained convex quadratic optimization problem. The solution of this problem maximizes the aggregate throughput subject to peak laser transmission power constraints. The optimization problem is solved in a closed form, and the resource allocation strategy is simple to implement in an optical network. Simulation results are presented, showing a total agreement between the derived analytical solution and the one obtained using a numerical search method. In addition, analytical and numerical results show that the proposed resource allocation strategy can offer substantial improvement in the system throughput.
\end{abstract}

Index Terms-Capacity, fiber Bragg grating (FBG), multirate, overlapped optical code division multiple access (OCDMA), overlapping coefficient, power control, quadratic function, rate control, throughput.

\section{INTRODUCTION}

$\mathbf{O}$ PTICAL CODE division multiple access (OCDMA) has received considerable attention as a multiple access scheme for optical local area networks [1], [2]. In addition, heterogeneous services, entailing multirate transmission, are now feasible due to the rapid evolution of fiber optic technology that offers ultrawide optical bandwidth that is capable of handling these multirate transmissions and fulfilling good quality-ofservice $(\mathrm{QoS})$ requirements.

The first work toward this target was presented in [3] and [4], where a novel coding technique that leads to the generation of a new family of optical orthogonal codes (OOCs) called the strict OOC. Although the strict OOC ensures both the autoand the cross correlation constraints to be less than or equal to one, the variability of transmission rate at constant power may not agree with the demanded QoS. Moreover, when the optical system attempts to transmit at a certain rate with very high power, a substantial increase in the interference occurs

Manuscript received August 3, 2006; revised March 7, 2007.

R. Raad and P. Fortier are with the Faculty of Science and Engineering, Laval University, Quebec City, QC G1K 7P4, Canada (e-mail: robert.raad.1@ ulaval.ca; fortier@gel.ulaval.ca).

E. Inaty is with the Faculty of Engineering, University of Balamand, El-Koura, Lebanon (e-mail: elie.inaty @ balamand.edu.lb).

H. M. H. Shalaby is with the Faculty of Engineering, University of Alexanderia, Alexanderia 21544, Egypt (e-mail: shalaby@ieee.org).

Color versions of one or more of the figures in this paper are available online at http://ieeexplore.iee.org.

Digital Object Identifier 10.1109/JLT.2007.901337 on the desired user at the receiver side [5]-[7]. This yields degradation in the system performance. One way to ameliorate the system efficiency is to adopt an efficient resource allocation strategy that regulates the amount of transmitted power with regard to the rate variation and the number of active users that maximizes the aggregate throughput of the multirate CDMA system [6].

Most of the analyses conducted on CDMA communication systems agree that optimal selection of the system's parameters such as the transmitted power and the bit rate would improve their performances [5]-[7]. This, in turn, gives rise to optimization problems that are rarely discussed in the literature of OCDMA. For instance, nonlinear programming power control algorithm has been proposed in [8] to maximize the capacity of multirate optical fast frequency hopping code division multiple access (OFFH-CDMA) system constrained by a predefined QoS based on the received signal-to-interference ratio (SIR) of each class of users. The rate of each class of users is chosen statically by choosing the corresponding processing gain (PG) in a way that higher rate users have smaller PG and lower rate users have larger PG. Then, the power of multirate users is optimally regulated with variable optical attenuator before transmission to limit the interference directly from the transmitter. In [9], a power control algorithm, based on optical power selector consisting of a set of optical hardlimiters and couplers, has been inspected for a multirate optical direct-sequence CDMA system using one signature for each user with time hopping. In this paper, the transmission rate and the bit error rate are controlled by the hopping rate and the optical power, respectively, to improve the system performance. Nevertheless, this algorithm employs ideal optical hardlimiters that are practically very difficult to realize. In addition, an adaptive overlapped pulseposition modulator, which is employed to create multirate and multiquality transmission schemes, has been investigated in [10] for OCDMA networks, where power control mechanism is done by means of an optical attenuators. It was proven that this system can tolerate four different services associated with four different classes of rates, which are easily differentiated by the intensity of the transmitted pulses and the number of transmitting slots, respectively. Moreover, the power control problem is also addressed in [11] for a temporal prime-coded OCDMA system taking into consideration the effect of the near-far problem caused by different fiber lengths connecting the users to the star network. It was shown that the fiber length after the star coupler is irrelevant to the optimal power evaluation. However, the consequences of multirate users were not investigated in this analysis. 
In this paper, and for the first time, we propose a novel hybrid power/rate control algorithm for overlapped OFFHCDMA (OOFFH-CDMA) system [12], [13] in which multirate transmission is achieved by overlapping consecutive bits while coded using fiber Bragg grating (FBG). It is shown in [12] that it is possible to increase the transmission of each class of users well beyond the nominal rate without decreasing the PG. In addition, a service curve has been introduced, which relates the cutoff rates of the offered multimedia classes in a multiclass system. Our purpose in this paper is to find the optimal overlapping coefficient through which we can achieve maximum transmission rate with minimum transmitted optical power directly from a laser source according to a predefined QoS required at the optical receivers for each class of users. In our analysis, we consider the average SIR as an adequate QoS requirement for each class of users. We derive an explicit solution of the optimal power as a function of the optimal data rate, from which the throughput function has been simplified to a quadratic function of the transmission rate vector. For each class of users, we provide a joint transmission power and overlapping coefficient allocation strategy, which has been obtained via the solution of a constrained optimization problem, which maximizes the aggregate system throughput subject to a peak laser transmission power constraint. Under this strategy, the classes of users are allocated maximum transmission rate in decreasing order of the QoS requirements. It is also shown that there is at most one class of users that has an overlapping coefficient between zero and full overlap, and the remaining classes either transmit with full overlap or with no overlap.

Following the introduction, this paper is structured as follows. Section II introduces the system model and the optimization problem formulation. The resource allocation problem is obtained in Section III. Section IV presents the solution for a two-class system. Results for a three-class system are covered in Section V. Finally, the conclusion is presented in Section VI.

\section{System Model and Problem Formulation}

An OOFFH-CDMA system that supports $M$ users in $S$ classes, sharing the same optical medium in a star architecture, has been proposed in [12]. We will consider that all users transmitting their data at the same QoS are clustered in the same class. All classes have the same PG $G$. The encoding-decoding is achieved passively using a sequence of FBGs. The gratings spectrally and temporarily slice the incoming broadband pulse into several components that are equally spaced at chip interval $T_{\mathrm{c}}$. The chip duration and the number of grating $G$ determine the nominal bit duration to be $T_{\mathrm{n}}=G T_{\mathrm{c}}$. The corresponding nominal transmission rate is $R_{\mathrm{n}}=1 / T_{\mathrm{n}}$. Increasing the transmission rate beyond the nominal rate $R_{\mathrm{n}}$ without decreasing $G$ introduces an overlapping of coefficient $\varepsilon_{j}$ among the transmitted bits during the same period $T_{\mathrm{n}}$, as revealed in Fig. 1 .

In this case, the concept of overlapping is illustrated among six bits of $G=5$, and the overlapping coefficient of class- $j$ is $\varepsilon_{j}=3$, which means that there are three chips in each OCDMA-coded bit that overlap with three chips of the other bits in the same class. This, in turn, augments the overall transmission rate of the users involved in this class from three bits

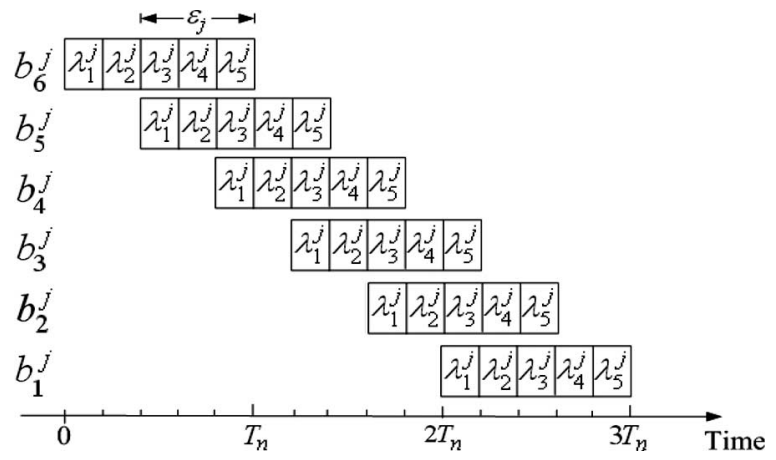

Fig. 1. Concept of overlapping among the bits of class- $j$ users, showing the effect of the overlapping coefficient $\varepsilon_{j}$ on their transmission rate.

after $3 T_{\mathrm{n}}$ to six bits. In general, the overlapping coefficient represents the number of overlapped chips among consecutive bits of class- $j$. Accordingly, the new transmission rate of class- $j$ is given by

$$
R_{j}=\frac{G}{G-\varepsilon_{j}} R_{\mathrm{n}}
$$

where $0 \leq \varepsilon_{j} \leq G-1$ for $j \in\{0, \ldots, S-1\}$. This implies that $R^{(\ell)} \leq R_{j} \leq R^{(u)}$, where $R^{(\ell)}=R_{\mathrm{n}}$ and $R^{(u)}=G R_{\mathrm{n}}$ are the lower and the upper data rate common to all classes, respectively. Also, we assume that the system is chip-synchronous and of discrete rate variation. Furthermore, all users of the same class transmit with the equal power and have the same overlapping coefficient. Hence, each class is characterized by its own QoS. Thus, let $P_{j}$ and $\beta_{j}$ be the transmitted power and QoS of class- $j$, respectively.

\section{A. SIR as QoS Measure}

In many cases, it is reasonable to take the QoS requirements as meeting the SIR constraints [6], [8]. It was shown in [12] that the SIR for class- $j$ using an OOFFH-CDMA system is given by

$$
\operatorname{SIR}_{j}=\frac{P_{j} G^{2}}{\frac{(M-1) G^{2}}{2 F} \sum_{i=0}^{S-1} \frac{p^{(i)} P_{i}}{G-\varepsilon_{i}}+\sigma_{\mathrm{n}}^{2}}, \quad j \in\{0, \ldots, S-1\}
$$

where $F$ is the total number of available frequencies used in the code construction [14], and $\sigma_{\mathrm{n}}^{2}$ is the variance of additive white Gaussian noise. On the other hand, $p^{(i)}$ is the multimedia probability density function and it represents the probability that a user selects class- $i$, where $\sum_{i=0}^{S-1} p^{(i)}=1$. We can easily simplify (2) into the following:

$$
\mathrm{SIR}_{j}=\frac{P_{j}}{\sum_{i=0}^{S-1} M_{i} P_{i} R_{i}+\frac{\sigma_{\mathrm{n}}^{2}}{G^{2}}}, \quad j \in\{0, \ldots, S-1\}
$$

where $R_{i}$ is given in (1), and $M_{i}$ represents the weight factor of class- $i$ and is given by

$$
M_{i}=\frac{M-1}{2 F G R_{\mathrm{n}}} p^{(i)} .
$$


Note that by increasing $p^{(i)}$ of class- $i$ as if we are increasing its weight for fixed system parameters $F, G, M$, and $R_{\mathrm{n}}$.

\section{B. System Throughput}

In this paper, we aim at finding an appropriate resource allocation strategy that maximizes the transmission rates, as well as minimizes the transmitted powers for each class of users in a multirate overlapped CDMA environment in a way to maximize the system capacity. The criterion to achieve this optimality is to consider the aggregate throughput $\Omega^{M}: \mathbb{R}^{S} \times$ $\mathbb{R}^{S} \rightarrow \mathbb{R}_{+}$as the weighted sum of the ratios of the transmission rates over transmitted powers for the $S$ classes, and it is given by

$$
\Omega^{M}(\mathbf{R}, \mathbf{P})=\sum_{j=0}^{S-1} M_{j} \frac{R_{j}}{P_{j}}
$$

where $\mathbf{R}=\left(R_{0}, R_{1}, \ldots, R_{S-1}\right)^{\mathrm{T}}$ is the data rate vector, $\mathbf{P}=$ $\left(P_{0}, P_{1}, \ldots, P_{S-1}\right)^{\mathrm{T}}$ is the power vector, and $M_{j}$ is the weight factor of class- $j$, as defined in (4) with $0 \leq M_{j} \leq 1$. This function of merit represents the system throughput, as the average number of bits per second per unit of power.

Accordingly, we are interested in computing the jointly optimal power and rate allocation for users in each class that maximizes the aggregate throughput, subject to predetermined QoS constraints in terms of the SIR of each class. The optimal allocation policy is obtained by solving the following optimization problem:

$$
\left(\Pi_{1}\right) \quad\left(\mathbf{R}^{*}, \mathbf{P}^{*}\right)=\arg \max _{(\mathbf{R}, \mathbf{P}) \in \Im}\left\{\Omega^{M}(\mathbf{R}, \mathbf{P})\right\}
$$

where the feasible set is given by

$$
\begin{aligned}
\Im=\{(\mathbf{R}, \mathbf{P}): & \operatorname{SIR}_{j}=\beta_{j}, 0<P_{j} \leq P_{\max } \text { and } \\
& \left.R_{\mathrm{n}} \leq R_{j} \leq G R_{\mathrm{n}}, \forall j \in\{0, \ldots, S-1\}\right\}
\end{aligned}
$$

where $P_{\max }<\infty$ is the maximum permissible power of the laser source, and $\beta_{j}$ is the QoS of class- $j$.

The nonlinearity of the optimization problem is obvious from (5) and (6). Thus, to solve $\left(\Pi_{1}\right)$, the problem is decoupled into two resource allocation scenarios: the power allocation scenario and the rate allocation scenario as will be shown in the next section. We first obtain the transmission power allocations, which are determined as a function of the QoS and the transmission rate of each class. We then maximize the aggregate throughput with respect to the transmission rate vector.

\section{Jointly Optimal Power and Rate Allocations}

In this scenario, we consider that the intensity of the transmitted optical signal is directly adjusted from the laser source with respect to the transmission data rate of users of the $S$ classes. Thus, each class is allocated the minimum optical power capable of handling the traffic rate of its users while observing the transmission rate of all other classes and at the same time maintaining a low level of interference at the desired receiver. To do so, we fix the transmission rate of all the classes, and we find out the optimal transmitted power corresponding to the desired class for a given QoS. Therefore, by taking $\mathrm{SIR}_{j}=\beta_{j}$ and rearranging terms in (3), we get a set of linear equality constraints in terms of $P_{j}$. That is

$\sum_{i=0}^{S-1} P_{i} M_{i} R_{i}-\frac{P_{j}}{\beta_{j}}+\frac{1}{\mathrm{SNR}_{\mathrm{n}}}=0, \quad \forall j \in\{0, \ldots, S-1\}$

where $\operatorname{SNR}_{\mathrm{n}}=\left(G^{2} / \sigma_{\mathrm{n}}^{2}\right)$ is the nominal signal-to-noise ratio common to all classes. Then, by solving the linear system in (7) for $P_{j}$, we get

$$
P_{j}=\frac{1}{\mathrm{SNR}_{\mathrm{n}}} \times \frac{\beta_{j}}{1-\sum_{i=0}^{S-1} \beta_{i} M_{i} R_{i}}, \quad \forall j \in\{0, \ldots, S-1\} .
$$

The power is defined when the denominator is strictly greater than zero. That is

$$
\sum_{i=0}^{S-1} M_{i} \beta_{i} R_{i}<1
$$

Consequently, the optimal class- $j$ transmission power $P_{j}^{*}$ is obtained by solving the rate allocation problem and finding the optimal rate $R_{j}^{*}$. Note that the thermal noise, dark current, and surface leakage current of the system are taken into consideration through the presence of the factor $\mathrm{SNR}_{\mathrm{n}}$ in the power allocation strategy.

\section{A. Optimal Rate Allocation}

In this scenario, we will compute the optimal rate of the system classes that corresponds to the minimum power obtained in the previous section by substituting $P_{j}$ in (8) into (5). We obtain

$$
\begin{aligned}
\Omega^{M}(\mathbf{R}) & =\mathbf{S N R}_{\mathrm{n}}\left(-\mathbf{R}^{\mathbf{T}} \mathbf{Q R}+\mathbf{C}^{\mathbf{T}} \mathbf{R}\right) \\
\mathbf{Q} & =\mathbf{Q}^{\mathbf{T}}, \quad \mathbf{Q}>0, \quad \mathbf{C}>0
\end{aligned}
$$

where $\mathbf{Q}_{S \times S}=\left[(1 / 2) M_{i} M_{j}\left(\left(\beta_{i} / \beta_{j}\right)+\left(\beta_{j} / \beta_{i}\right)\right)\right]_{i, j=0,1 \ldots, S-1}$, and $\mathbf{C}=\left[\left(M_{0} / \beta_{0}\right)\left(M_{1} / \beta_{1}\right) \cdots\left(M_{S-1} / \beta_{S-1}\right)\right]^{\mathrm{T}}$. Notice that the throughput function is a quadratic function of the rate vector $\mathbf{R}$.

Thus, the optimization problem $\left(\Pi_{1}\right)$ under the optimal power allocation becomes

$$
\left(\Pi_{2}\right) \quad \mathbf{R}^{*}=\arg \max _{\mathbf{R} \in \Im}\left\{\Omega^{M}(\mathbf{R})\right\}
$$

where the feasible set $\Im$ is given by

$$
\begin{gathered}
\Im=\left\{\mathbf{R}: \quad \sum_{j=0}^{S-1} M_{j} \beta_{j} R_{j} \leq 1-\frac{\max \left(\beta_{j}\right)_{j=0,1, \ldots, S-1}}{P_{\max } \mathrm{SNR}_{\mathrm{n}}}\right. \\
\left.\quad \text { and } R_{\mathrm{n}} \leq R_{j} \leq G R_{\mathrm{n}}, \forall j \in\{0, \ldots, S-1\}\right\} .
\end{gathered}
$$


Notice that the gradient of $\Omega^{M}(\mathbf{R})$ can be computed as

$$
\nabla \Omega^{M}=\operatorname{SNR}_{\mathrm{n}}(-2 \mathbf{Q R}+\mathbf{C})
$$

and the Hessian matrix [15], [16] is

$$
\mathbf{H}=\nabla^{2} \Omega^{M}=-2 \mathrm{SNR}_{\mathrm{n}} \mathbf{Q} .
$$

Because the Hessian matrix is negative, the throughput function is a concave function in $\mathbf{R}$, and therefore, the Kuhn-Tucker (KT) condition [16] is sufficient for an optimal point to be a maximum. To solve $\left(\Pi_{2}\right)$, we use the method of Lagrange multiplier. Consequently, the Lagrangian function is defined as

$$
L(\mathbf{R}, \boldsymbol{\Lambda})=\Omega^{M}(\mathbf{R})+\sum_{m=0}^{2 S} \lambda_{m} g_{m}
$$

where $g_{m}$ is the $m$ th constraint, $\lambda_{m}$ is the corresponding Lagrangian multiplier, and $\boldsymbol{\Lambda}$ is the vector of Lagrangian multipliers. Applying the KT condition on $\left(\Pi_{2}\right)$, we obtain

$$
\begin{aligned}
\frac{\partial L(\mathbf{R}, \boldsymbol{\Lambda})}{\partial R_{j}} & =0, \quad \forall j \in\{0, \ldots, S-1\} \\
\lambda_{m} g_{m} & =0, \quad \forall m \in\{0, \ldots, 2 S\} \\
g_{m} & \geq 0 \\
\lambda_{m} & \geq 0 .
\end{aligned}
$$

The nature of the stationary points is governed by the secondorder derivative of the Lagrangian function [15]. Notice that the second-order derivative is strictly negative and independent of $R_{j}$, i.e.,

$$
\frac{\partial^{2} L(\mathbf{R}, \boldsymbol{\Lambda})}{\partial R_{j}^{2}}=-2 \mathrm{SNR}_{\mathrm{n}} M_{j}^{2}
$$

and

$$
\frac{\partial^{2} L(\mathbf{R}, \boldsymbol{\Lambda})}{\partial R_{i} \partial R_{j}}=-\mathrm{SNR}_{\mathrm{n}} M_{i} M_{j}\left(\frac{\beta_{i}}{\beta_{j}}+\frac{\beta_{j}}{\beta_{i}}\right), \quad i \neq j .
$$

This implies that (20) and (21) are sufficient conditions for the stationary points to be maxima [16]. The following two propositions show that the global maximum of $\Omega^{M}(\mathbf{R})$ is not the solution of $\left(\Pi_{2}\right)$.

Proposition 1: Given an $S \times S$ positive symmetric matrix $\mathbf{Q}$ of the form

$$
\mathbf{Q}_{S \times S}=\left[\frac{1}{2} M_{i} M_{j}\left(\frac{\beta_{i}}{\beta_{j}}+\frac{\beta_{j}}{\beta_{i}}\right)\right]_{\forall i, j \in\{0,1, \ldots, S-1\}}
$$

where all $\beta_{i} \neq \beta_{j}, \mathbf{Q}$ is nonsingular for $S=2$, and it is singular for $S \geq 3$.

Proof: Let $\mathbf{A}_{2 \times 2}$ be any $2 \times 2$ matrix of the form

$\mathbf{A}_{2 \times 2}$

$$
=\left[\begin{array}{cc}
\frac{1}{2} M_{i} M_{k}\left(\frac{\beta_{i}}{\beta_{k}}+\frac{\beta_{k}}{\beta_{i}}\right) & \frac{1}{2} M_{i} M_{\ell}\left(\frac{\beta_{i}}{\beta_{\ell}}+\frac{\beta_{\ell}}{\beta_{i}}\right) \\
\frac{1}{2} M_{i+1} M_{k}\left(\frac{\beta_{i+1}}{\beta_{k}}+\frac{\beta_{k}}{\beta_{i+1}}\right) & \frac{1}{2} M_{i+1} M_{\ell}\left(\frac{\beta_{i+1}}{\beta_{\ell}}+\frac{\beta_{\ell}}{\beta_{i+1}}\right)
\end{array}\right]
$$

where $i=S-2, S \geq 2$, and $k \neq \ell, k, \ell \in\{0,1, \ldots, S-1\}$.

Then, the determinant of $\mathbf{A}_{2 \times 2}$ is equal to

$\operatorname{det}\left(\mathbf{A}_{2 \times 2}\right)$

$$
=-\frac{1}{4} M_{i} M_{i+1} M_{k} M_{\ell}\left(\frac{\beta_{i}}{\beta_{i+1}}-\frac{\beta_{i+1}}{\beta_{i}}\right)\left(\frac{\beta_{k}}{\beta_{\ell}}-\frac{\beta_{\ell}}{\beta_{k}}\right) \neq 0
$$

given that all $\beta \mathrm{s}$ are distinct. This implies that the matrix is nonsingular. For $S=2, \mathbf{Q}_{2 \times 2}$ is a special case of $\mathbf{A}_{2 \times 2}$, where $i=0, k=0$, and $\ell=1$. Hence, $\mathbf{Q}_{2 \times 2}$ is symmetric and nonsingular.

Now, Let $\mathbf{A}_{3 \times 3}$ be any $3 \times 3$ matrix of the form shown at the bottom of the page, where $i=S-3, S \geq 3$, and $k \neq \ell \neq$ $r, k, \ell, r \in\{0,1, \ldots, S-1\}$.

Then, $\operatorname{det}\left(\mathbf{A}_{3 \times 3}\right)=0$. This implies that this matrix is singular. Consequently, all the $3 \times 3$ minors in the determinant of $\mathbf{Q}_{S \times S}$ are zeros; hence, $\operatorname{det}\left(\mathbf{Q}_{S \times S}\right)=0$ and $\mathbf{Q}_{S \times S}$ is singular for $S \geq 3$.

Proposition 2: The global maximum of the optimization problem $\left(\Pi_{2}\right)$ is not feasible.

Proof: The throughput function has a global maximum only when the gradient is null, $\nabla \Omega^{M}=0$. This implies

$$
\mathbf{R}^{*}=\frac{\mathbf{Q}^{-1} \mathbf{C}}{2} .
$$

The global maximum in (22) exists if and only if the matrix $\mathbf{Q}$ is invertible. In addition, $\mathbf{R}^{*}$ is feasible if it is in the feasible set $\Im$ of $\left(\Pi_{2}\right)$. By proposition $1, \mathbf{Q}$ is nonsingular for $S=2$. Thus, for any two-class system, for example class- $i$ and class- $j$, the global maximum is found when the gradient is null. That is $\nabla \Omega^{M}=0$, which yields

$$
\begin{aligned}
R_{i}^{*} & =\frac{\beta_{i}}{M_{i}\left(\beta_{i}^{2}-\beta_{j}^{2}\right)} \\
R_{j}^{*} & =\frac{\beta_{j}}{M_{j}\left(\beta_{j}^{2}-\beta_{i}^{2}\right)} .
\end{aligned}
$$

By assumption, $\beta_{i} \neq \beta_{j}$, so both rates are finite. Also, notice that, $\beta_{i} M_{i} R_{i}+\beta_{j} M_{j} R_{j}=1$, which violates the condition in (9). This means that this solution is not feasible and, hence, the

$$
\mathbf{A}_{3 \times 3}=\left[\begin{array}{ccc}
\frac{1}{2} M_{i} M_{k}\left(\frac{\beta_{i}}{\beta_{k}}+\frac{\beta_{k}}{\beta_{i}}\right) & \frac{1}{2} M_{i} M_{\ell}\left(\frac{\beta_{i}}{\beta_{\ell}}+\frac{\beta_{\ell}}{\beta_{i}}\right) & \frac{1}{2} M_{i} M_{r}\left(\frac{\beta_{i}}{\beta_{r}}+\frac{\beta_{r}}{\beta_{i}}\right) \\
\frac{1}{2} M_{i+1} M_{k}\left(\frac{\beta_{i+1}}{\beta_{k}}+\frac{\beta_{k}}{\beta_{i+1}}\right) & \frac{1}{2} M_{i+1} M_{\ell}\left(\frac{\beta_{i+1}}{\beta_{\ell}}+\frac{\beta_{\ell}}{\beta_{i+1}}\right) & \frac{1}{2} M_{i+1} M_{r}\left(\frac{\beta_{i+1}}{\beta_{r}}+\frac{\beta_{r}}{\beta_{i+1}}\right) \\
\frac{1}{2} M_{i+2} M_{k}\left(\frac{\beta_{i+2}}{\beta_{k}}+\frac{\beta_{k}}{\beta_{i+2}}\right) & \frac{1}{2} M_{i+2} M_{\ell}\left(\frac{\beta_{i+2}}{\beta_{\ell}}+\frac{\beta_{\ell}}{\beta_{i+2}}\right) & \frac{1}{2} M_{i+2} M_{r}\left(\frac{\beta_{i+2}}{\beta_{r}}+\frac{\beta_{r}}{\beta_{i+2}}\right)
\end{array}\right]
$$


global maximum. For $S \geq 3$, $\mathbf{Q}$ is singular by Proposition 1 , and therefore, the global maximum does not exist.

\section{B. Problem Solution}

The solution of $\left(\Pi_{2}\right)$ is obtained via the following lemmas, the first of which shows that the feasible set $\Im$ can be reduced to its boundaries. It also shows that there is at most one class of users that has an overlapping coefficient between zero and full overlap, and the remaining classes either transmit with full overlap or with no overlap.

Lemma 1: Assume that $\mathbf{R}^{*}=\left(R_{0}^{*}, R_{1}^{*}, \ldots, R_{S-1}^{*}\right)^{\mathrm{T}}$ solves the optimization problem $\left(\Pi_{2}\right)$. If $P_{\mathrm{Max}}<\infty$, there exists at most one $R_{j}^{*}$ such that $R^{(\ell)} \leq R_{j}^{*} \leq R^{(u)}$, and $R_{i}^{*}=R^{(u)}$ or $R_{i}^{*}=R^{(\ell)} \forall i \neq j=0,1, \ldots, S-1$.

Proof: The throughput function is concave in $\mathbf{R}^{*}$ and thus, the optimal rate allocation lies on the boundary of the feasible set $(\Im)$. Consider the KT conditions on $\left(\Pi_{2}\right) \forall j \in$ $\{0,1, \ldots, k, \ldots, S-1\}$ as follows:

$$
\begin{aligned}
\frac{\partial \Omega^{M}}{\partial R_{j}}+\lambda_{j}-\lambda_{S+j}-\lambda_{2 S} M_{j} \beta_{j} & =0 \\
\lambda_{j}\left(R_{j}-R^{(\ell)}\right) & =0 \\
\lambda_{S+j}\left(R^{(u)}-R_{j}\right) & =0 \\
\lambda_{2 S}\left(1-\frac{\max \left(\beta_{j}\right)_{\forall j \in\{0,1, \ldots, S-1\}}}{P_{\operatorname{Max}} \mathrm{SNR}_{\mathrm{n}}}-\sum_{i=0}^{S-1} M_{i} \beta_{i} R_{i}\right) & =0 .
\end{aligned}
$$

There are four cases to consider.

1) If $\lambda_{j} \neq 0$, then $\lambda_{S+j}=0$. This implies that $R_{j}^{*}=R^{(\ell)}$, $\forall j \in\{0,1, \ldots, k, \ldots, S-1\}$.

2) If $\lambda_{S+j} \neq 0$, then $\lambda_{j}=0$. This implies that $R_{j}^{*}=R^{(u)}$, $\forall j \in\{0,1, \ldots, k, \ldots, S-1\}$.

In both cases, there are $(S+1)$ equations of $(S+1)$ unknowns in $\lambda_{j}$ and/or $\lambda_{S+j}$ plus $\lambda_{2 S}$. If $\lambda_{2 S} \neq 0$, or $\lambda_{2 S}=0$ then

$$
\sum_{i=0}^{S-1} M_{i} \beta_{i} R_{i}^{*} \leq\left(1-\frac{\max \left(\beta_{j}\right)_{\forall j \in\{0,1, \ldots, S-1\}}}{P_{\operatorname{Max} \mathrm{SNR}_{\mathrm{n}}}}\right)
$$

which satisfies condition (9). Therefore, $R_{j}^{*}$ are indeed feasible solutions for $\left(\Pi_{2}\right)$.

3) Consider the class- $k$ of users for which $\lambda_{k}=0$ and $\lambda_{S+k}=0$. This implies that the feasible solution is $R_{k}^{*} \neq$ $\left\{R^{(\ell)}, R^{(u)}\right\}$. In addition to this condition, if cases 1 ) and/or 2) are occurring for all $j \neq k$, there are also $(S+1)$ equations of $(S+1)$ unknowns in $\lambda_{j \neq k}$ and/or $\lambda_{S+j \neq k}$ plus $\lambda_{2 S}$ and $R_{k}$. If $\lambda_{2 S} \neq 0, R_{k}^{*}$ is computed from constraint (26), and it is given by

$R_{k}^{*}=\frac{1}{M_{k} \beta_{k}}\left\{\left(1-\frac{\max \left(\beta_{j}\right)_{\forall j \in\{0,1, \ldots, S-1\}}}{P_{\operatorname{Max}_{1} \mathrm{SNR}_{\mathrm{n}}}}\right)-\sum_{\substack{i=0 \\ \neq k}}^{S-1} M_{i} \beta_{i} R_{i}^{*}\right\}$

where $R_{i}^{*} \in\left\{R^{(l)}, R^{(u)}\right\}$.

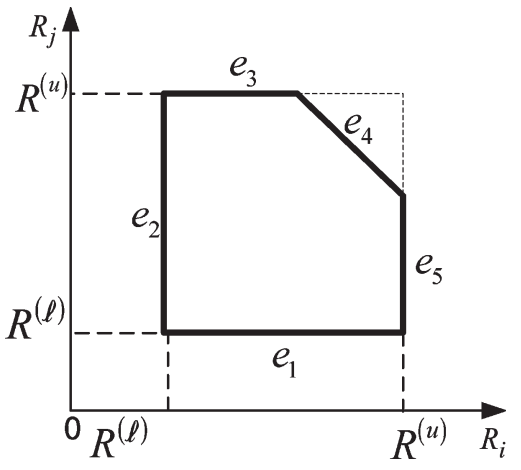

Fig. 2. Feasible region for a two-class system.

In addition, $\lambda_{2 S}$ is computed from (23). On the other hand, if $\lambda_{2 S}=0$, then

$$
\sum_{i=0}^{S-1} M_{i} \beta_{i} R_{i}^{*} \leq\left(1-\frac{\max \left(\beta_{j}\right)_{\forall j \in\{0,1, \ldots, S-1\}}}{P_{\operatorname{Max}} \mathrm{SNR}_{\mathrm{n}}}\right)
$$

because $R_{i}^{*}$ are feasible by assumption. In this case, $R_{k}^{*}$ is computed from $\left(\partial \Omega^{M} / \partial R_{k}\right)=0$.

Since, by assumption, $R_{k}^{*}$ is feasible, $R^{(\ell)} \leq R_{k}^{*} \leq$ $R^{(u)}$, where all $R_{j}^{*}=R^{(\ell)}$ or $R^{(u)}, \forall j \neq k$.

4) If $\lambda_{j}=0$ and $\lambda_{S+j}=0$ for more than one class at the same time, then the system has no feasible solution by Proposition 1.

This means that there exists at most one class of users that transmits with rate between $R_{\mathrm{n}}$ and $G R_{\mathrm{n}}$, and the remaining classes either transmit with the maximum rate $G R_{\mathrm{n}}$ or with the minimum rate $R_{\mathrm{n}}$.

Lemma 2: Consider that $\beta_{i}>\beta_{j}, \forall i<j$. If $\mathbf{R}^{*}=$ $\left(R_{0}^{*}, R_{1}^{*}, \ldots, R_{S-1}^{*}\right)^{\mathrm{T}}$ solves the optimization problem $\left(\Pi_{2}\right)$, then $R_{i}^{*} \leq R_{j}^{*}$ if and only if $0 \leq i<j \leq S-1$.

Proof: See the Appendix.

\section{Two-Class SYSTEM}

In this section, we analyze the feasible region of the two-class system by providing a graphical representation of the system's solution. Thereafter, we proceed on by a numerical analysis to assess the validity of the optimal results.

\section{A. Feasible Region Analysis}

Due to the complexity of the problem under consideration, and without loss of generality, we present the case of a twoclass system. Consider a two-class system, the class- $i$ and the class- $j$ with $\mathbf{R}=\left(R_{i}, R_{j}\right)^{\mathrm{T}}$. The boundaries of the feasible region is illustrated in Fig. 2. To solve $\left(\Pi_{2}\right)$, we should obtain the optimal solution which is defined in predetermined intervals of $\beta_{i}$ and $\beta_{j}$. Let the set of edges of the feasible region be $\mathbf{E}=\left\{e_{1}, e_{2}, e_{3}, e_{4}, e_{5}\right\}$ to be the locus of our optimal solution. By Lemma 1, we know that, at most, one class of users transmits with transmission rate between $R^{(\ell)}$ and $R^{(u)}$, and the remaining classes either transmit with $R^{(u)}$ or with $R^{(\ell)}$. Thus, the search space of the optimal solution is $\mathbf{E}^{\prime}=\left\{e_{1}, e_{2}, e_{3}, e_{5}\right\}$. In addition, without loss of generality, consider that $\beta_{i}>\beta_{j}$. 


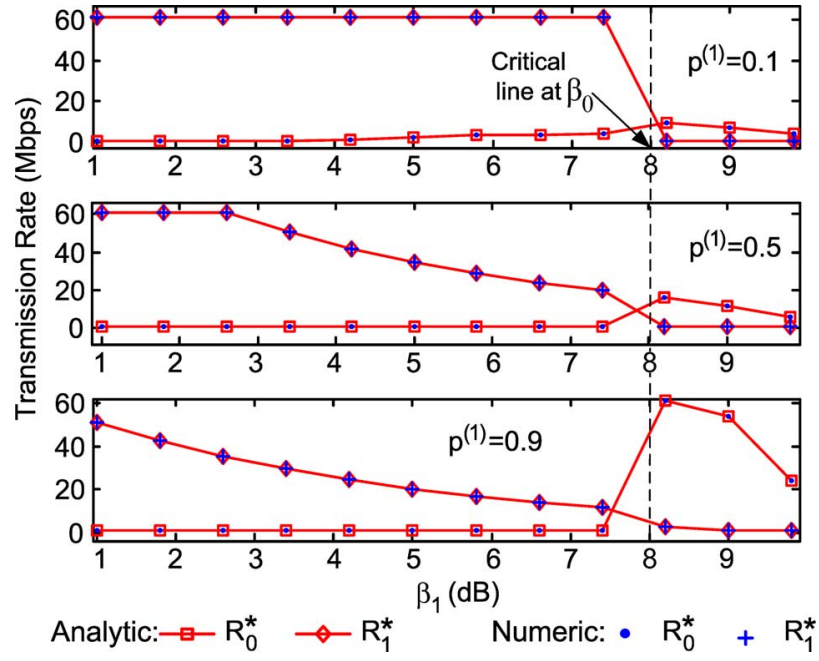

Fig. 3. Transmission rates versus QoS of class-1 users for different multimedia distribution.

By Lemma 2, we know that if $\beta_{i}>\beta_{j}$, then $R_{i} \leq R_{j}$. This means that the locus of the optimal solution has been reduced to $\mathbf{E}^{\prime \prime}=\left\{e_{2}, e_{3}\right\}$.

\section{B. Numerical Results}

In this part, we evaluate the effectiveness of the proposed power/rate control algorithm for two-class. First of all, we consider the two-class system for which we assume that $M=$ 61 users are active, the PG of the user's signature is $G=61$, the total number of available wavelengths is $F=62$, the nominal signal-to-noise ratio is $\mathrm{SNR}_{\mathrm{n}}=35.7 \mathrm{~dB}$, the upper bound on the laser power is $P_{\max }=5 \mathrm{dBm}$, the QoS of class- 0 is fixed to $\beta_{0}=8 \mathrm{~dB}$, and the nominal transmission rate is $R_{\mathrm{n}}=1 \mathrm{Mb} / \mathrm{s}$. Besides, in order to assess the validity of our results, we make use of a numerical method consisting of a sequential quadratic method, based on the quasi-Newton method, in which a quadratic programming subproblem is solved at each iteration, and an estimate of the Hessian of the Lagrangian function is updated using the BFGS method (as suggested by Broyden et al. in 1970) [16]. The simulation shows that the explicit analytic solution is completely matching the numeric one. This means that the derived solution is indeed the exact optimal solution. In addition, the performance of the proposed resource allocation strategy is compared with that of a classical power control algorithm with fixed transmission rates. If we assume that class0 users transmit at rate $R_{0} \cong 2 R_{1}$ when $\beta_{0}<\beta_{1}$ and class1 users transmit at rate $R_{1} \cong 2 R_{0}$ when $\beta_{1}<\beta_{0}$, then the classical power control strategy allocates the best transmission laser power to each user in either classes in order to guarantee the QoS requirements.

In Fig. 3, the transmission rates are evaluated as a function of $\beta_{1}$, and they are plotted for different multimedia distributions. For $p^{(1)}=0.1$, the multimedia traffic is more dense in class- 0 rather than in class-1. Since small number of users are choosing class-1, the minority class- 1 users transmit at rate $R_{1}^{*}=R^{(u)}$ for $\beta_{1}<8 \mathrm{~dB}$. On the other hand, the majority class- 0 users transmit at rate $R_{0}^{*}=R^{(\ell)}$ for $\beta_{1}<4 \mathrm{~dB}$, and $R^{(\ell)}<R_{0}^{*}<$ $R^{(u)}$ for $4 \mathrm{~dB}<\beta_{1}<8 \mathrm{~dB}$. In addition, for $\beta_{1}>8 \mathrm{~dB}$, class- 1

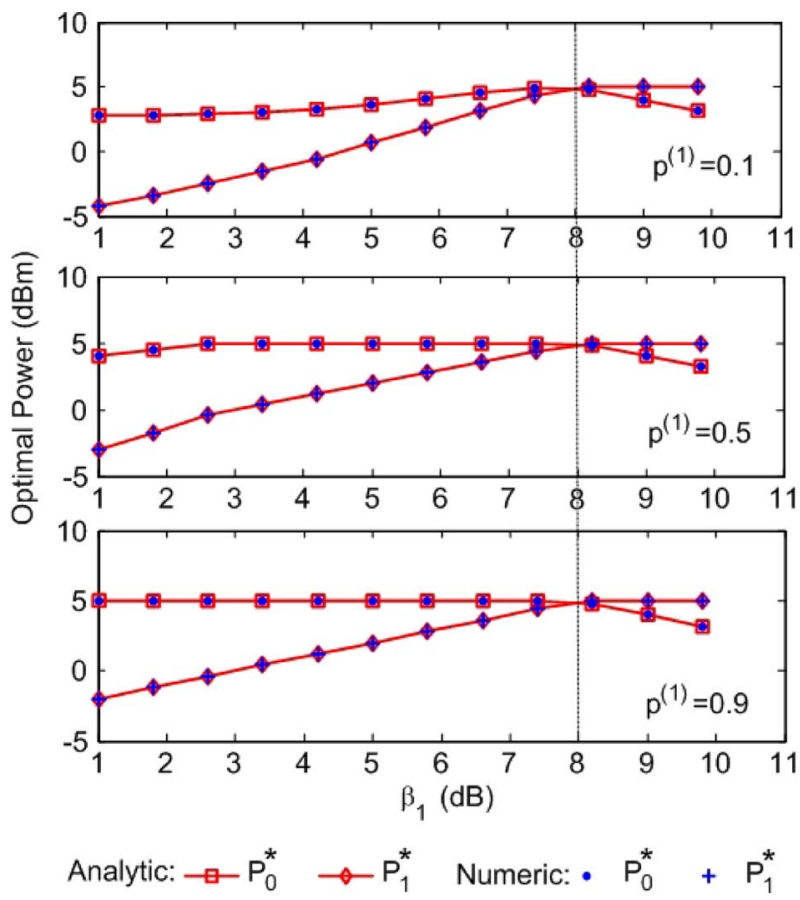

Fig. 4. Power consumption of class- 0 and class- 1 for different multimedia distributions.

users now transmit at rate $R_{1}^{*}=R^{(\ell)}$, while class-0 users transmit at rate $R^{(\ell)}<R_{0}^{*}<R^{(u)}$. For $p^{(1)}=0.9$, class- 1 users are allowed to transmit only at $R^{(\ell)}<R_{1}^{*}<R^{(u)}$ for $\beta_{1}<$ $8 \mathrm{~dB}$, while class- 0 users transmit at $R^{(\ell)}$. Also, notice that for $8 \mathrm{~dB}<\beta_{1}<9 \mathrm{~dB}, R^{(\ell)}<R_{1}^{*}<R^{(u)}$, and $R_{0}^{*}=R^{(u)}$. This, in turn, shows a total agreement with the hypotheses proposed in the two lemmas proven in previous sections. Furthermore, as the number of users in a certain class increases and, hence, the multimedia traffic, the allocated transmission rate decreases in order to keep the MAI at an acceptable level for satisfying the required QoS.

The optimal transmission power for the corresponding multimedia distributions is illustrated in Fig. 4. Note that, as the number of class- 0 users decreases, their allowable transmission power increases. Therefore, when $p^{(1)}$ increases from 0.1 to 0.9 , the MAI effect of class- 0 on class- 1 decreases, and the system allows class-0 to transmit at the upper bound laser power to improve the service requirement. In addition, class-1 power is proportional to QoS. Therefore, it is monotonically increasing as $\beta_{1}$ until it reaches a constant level at the maximum attainable laser power for $\beta_{1}>\beta_{0}$. The constant power in this interval of $\beta_{1}$ is necessary to assure the data transmission at such QoS. Further, we remark that an additional augmentation of $\beta_{1}$ above $10 \mathrm{~dB}$ is no longer supportable because the laser power of the source becomes inadequate.

The optimal throughput is plotted in Fig. 5 and compared to the nonoptimal one achieved by the classical power control strategy. The optimal throughput decays as $\beta_{1}$ increases because at high QoS, the allocated resources are performed to preserve the QoS requirement rather than to increase the system capacity. In addition, we observe that an appropriate traffic distribution gradually enhances the system throughput according to the allocated resources. For $p^{(1)}=0.1$, both $R_{0}^{*}$ 


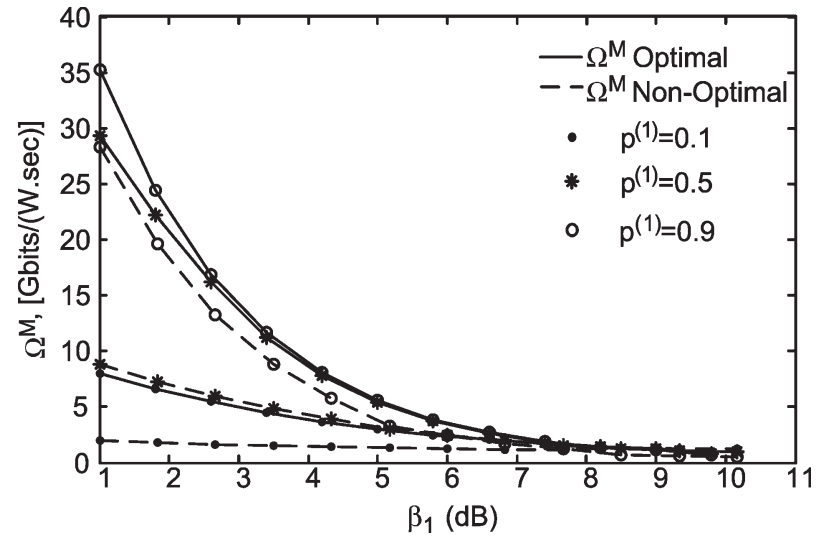

Fig. 5. Throughput versus QoS of class-1 for different multimedia distributions.

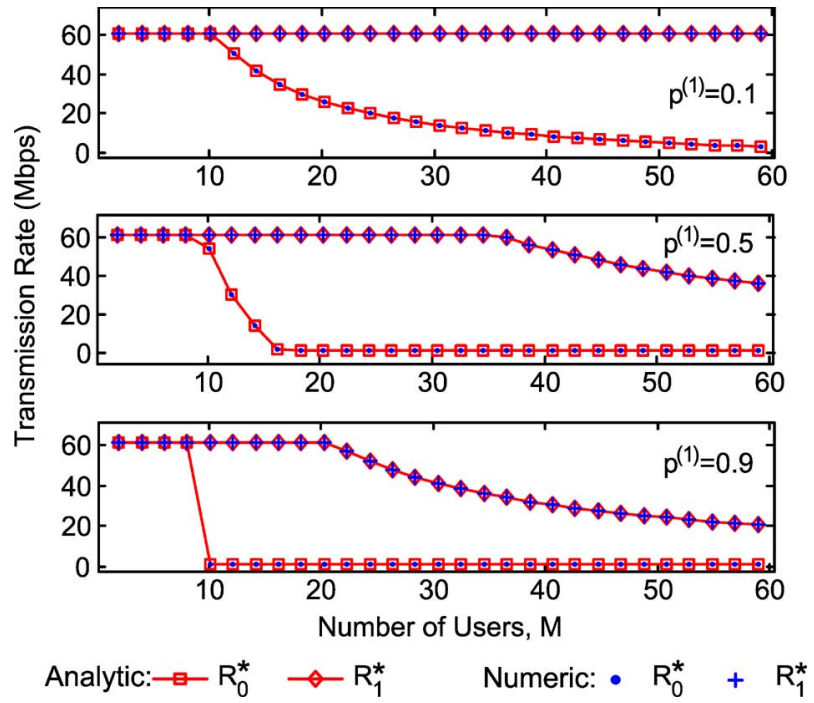

Fig. 6. Optimal transmission rates versus the number of users for a two-class system.

and $R_{1}^{*}$ are high, hence, the interference. Alongside, the allowable transmission power is relatively low. This yields the lowest optimal throughput due to the fact that the allocated power is insufficient to satisfy the QoS requirement and to combat the MAI increase. As $p^{(1)}$ increases, the MAI relaxes and the average system throughput increases. Also, we can clearly observe that the system throughput of the proposed resource allocation strategy is superior to that of the nonoptimal one for the different multimedia distributions.

Next, we study the effectiveness of the proposed resource allocation strategy with respect to the number of stations accessing the system for different multimedia distributions. We keep the same parameter settings as in the previous part, but in this case, we fix QoS of class- 1 to $\beta_{1}=5 \mathrm{~dB}$, which is $3 \mathrm{~dB}$ less than $\beta_{0}$. By this setting, the classical power control criteria with constant transmission rate turns out to be the equal energy criteria (EEC). We show that the EEC results are feasible but nonoptimal.

The optimal transmission rates in terms of $M$ are examined in Fig. 6. Consequently, for small $M$, the system allows both classes to transmit at maximum rate $R^{(u)}$ because the MAI is sufficiently small. However, when $M \geq 10$, as the user popula-
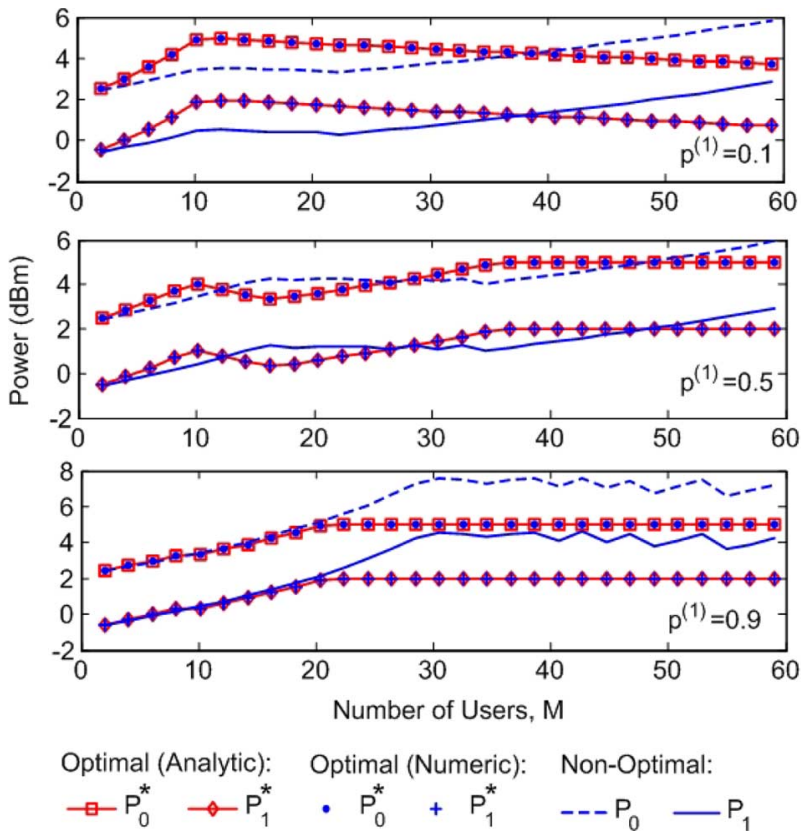

Fig. 7. Transmission power versus the number of users for a two-class system.

tion in a given class increases, its transmission rate decreases to keep the MAI at regular level and to accommodate to the QoS requirement. Note that, when $R^{(\ell)} \leq R_{0}^{*}<R^{(u)}, R_{1}^{*}=R^{(u)}$, and when $R_{0}^{*}=R^{(\ell)}, R^{(\ell)}<R_{1}^{*} \leq R^{(u)}$ is, thus, consistent with Lemma 1. Furthermore, because $\beta_{0}>\beta_{1}$, it is clear that we always have $R_{0}^{*} \leq R_{1}^{*}$, which validates Lemma 2 .

Recall that, our objective is to determine the optimal overlapping coefficient $\varepsilon_{j}^{*}$, which is a point on the service curve proposed in [12]. By obtaining the optimal rates $R_{0}^{*}$ and $R_{1}^{*}$ for both classes, the optimal overlapping coefficients $\varepsilon_{0}^{*}$ and $\varepsilon_{1}^{*}$ satisfying the system requirements are now computed using (1).

In Fig. 7, the optimal power is compared to the nonoptimal one as $M$ varies. Notice that when $p^{(1)}$ is small, the EEC allocates less power for small $M$ and more power for large $M$ compared to our newly proposed algorithm. This criterion makes the users always susceptible to MAI. In contrast, our proposed strategy provides more power for small $M$ to improve the optical signal and less power for large $M$ to reduce the MAI intensity. On the other hand, when $p^{(1)}=0.5$, the EEC follows the optimal one. Finally, when $p^{(1)}=0.9$, the EEC power is matching the optimal one for small $M$. However, it exceeds the upper bound laser power for large $M$. On the other hand, our proposed strategy controls this excess of power by clamping it to the maximum allowable laser power.

The impact of the total number of users on the system throughput is shown in Fig. 8. For small $M$, the performance of the EEC approaches the optimal one, particularly when the probability of selecting class-1 is high. As $M$ increases, the system throughput of our proposed strategy outperforms that of the EEC. Note that as $M$ becomes higher, the system throughput mounts for increasing values of $p^{(1)}$. This happens because when $p^{(1)}$ is small, both classes transmit at rates higher than the nominal one as revealed in Fig. 3. This in turn requires high overlapping coefficients. It follows that the interference level in the optical channel increases. In addition, the 


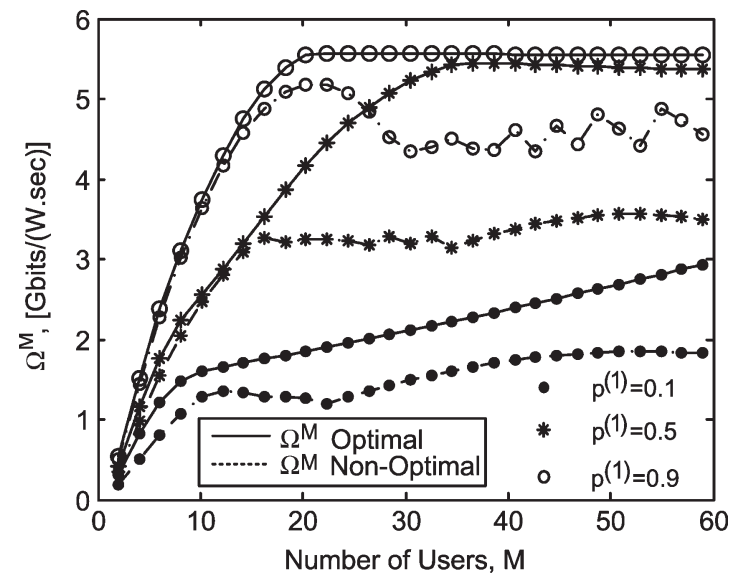

Fig. 8. Optimal throughput versus the total number of users for a two-class system.

transmission power level dedicated for such rate is also low, as shown in Fig. 4. This creates degradation in the system throughput. When $p^{(1)}$ increases, the transmission rates decrease, whereas the power relatively increases. Consequently, the throughput is significantly ameliorated.

\section{Three-Class System Numerical Evaluation}

Since our algorithm is derived for any number of multimedia classes, let us consider the case of a three-class system. Consider the three classes class- 0 , class- 1 , and class- 2 , among which the users are equally distributed, i.e., $p^{(0)}=p^{(1)}=$ $p^{(2)}=1 / 3$. Each class is characterized by its own QoS such that $\beta_{0}=11 \mathrm{~dB}, \beta_{1}=8 \mathrm{~dB}$, and $\beta_{2}=5 \mathrm{~dB}$, respectively. Without loss of generality, the classes are rearranged by descending order of QoS, such that the additional class comes first. Let $P_{\mathrm{Max}}=8 \mathrm{dBm}$ to be the maximum laser power. In order to respect the EEC power settings, notice that there are 3-dB differences between each two consecutive QoSs, and the transmission rates of the three classes are set to be $R_{2} \cong 2 R_{1}$ and $R_{1} \cong 2 R_{0}$.

The three-class optimal transmission rates versus $M$ are illustrated in Fig. 9. It is clear that only one class can transmit at rate between $R^{(\ell)}$ and $R^{(u)}$, and the other classes either at $R^{(\ell)}$ or $R^{(u)}$. This completely conveys with Lemma 1. Also, note that $R_{0}^{*} \leq R_{1}^{*} \leq R_{2}^{*}$ as $\beta_{0}>\beta_{1}>\beta_{2}$, which confirms the validity of Lemma 2 for the three-class system as well.

The effect of adding a third class, with high QoS, to the multirate system on the optimal transmission laser power has been inspected in Fig. 10. As $M$ increases, the power increases to stabilize to $8 \mathrm{dBm}$. In addition, the upper bound powers of class- 1 and class- 2 have not changed since the two-class system, even though their overall allocated powers have been slightly diminished with 3-dBm differences between each two consecutive classes. This is caused by the reduction in the user's population in each of the three classes and the augmentation of the total optical power in the fiber link. Furthermore, the power provided by EEC is not properly allocated. Therefore, less power is allocated for small number of users $(M<20)$, while more power is allocated for large number of users $(M>20)$.

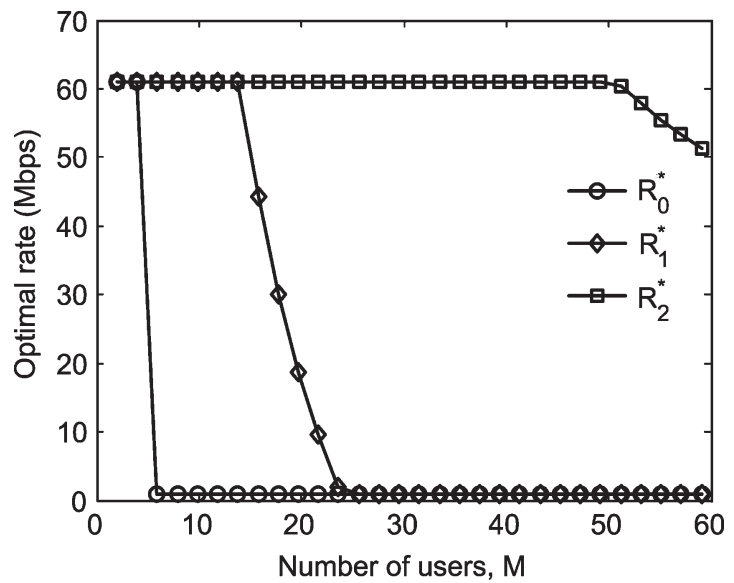

Fig. 9. Optimal transmission rates versus the number of users for a three-class system.

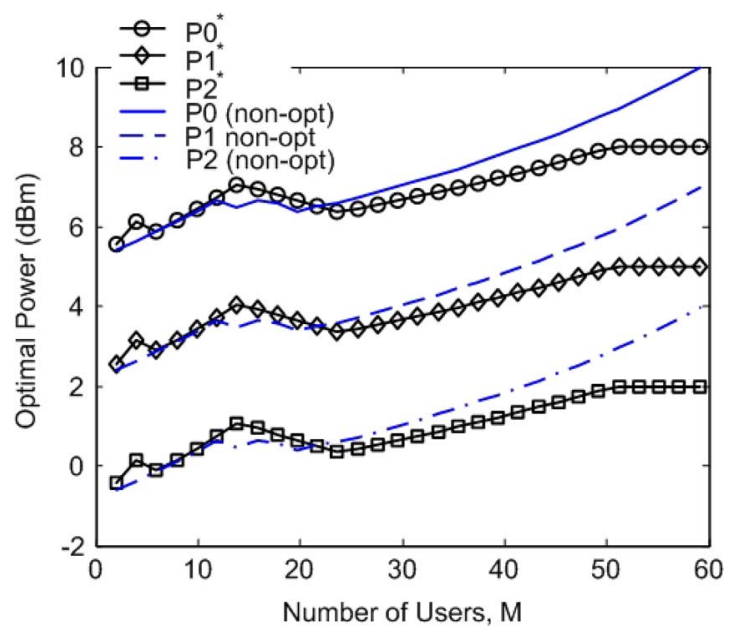

Fig. 10. Transmission power versus the number of users for a three-class system.

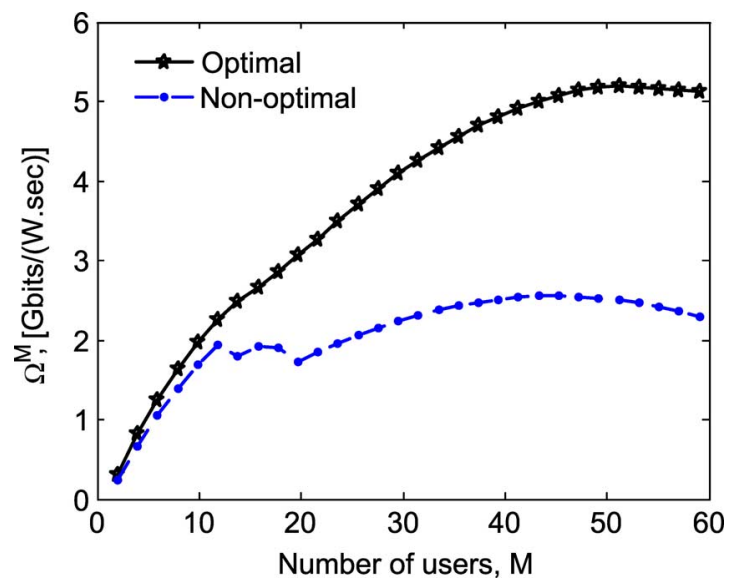

Fig. 11. Optimal throughput versus the total number of users for a three-class system.

The throughputs of both strategies are envisaged in Fig. 11. Notice that the performance of our proposed strategy is always superior to that of EEC. 


\section{CONCLUSION}

A new resource allocation strategy was proposed for OOFFH-CDMA system. With this strategy, the system throughput is the average successfully transmitted bits per second per unit of power. However, due to the nonlinearity of the throughput and the constraint functions and, hence, the optimization problem, two recourse allocation scenarios were derived for both power and rate to simplify the analysis. Then, KT theorem had been applied on the rate allocation scenario to find out the optimal transmission rates upon which the optical intensity of each class was optimally regulated directly form the laser source. It was proven that this system, in general, has no global maximum but a local maximum for a given set of QoSs. This local maximum has as coordinates the transmission rates of the $S$ classes satisfying Lemmas 1 and 2. Afterward, twoand three-class systems had been simulated as particular case studies. The simulation assessed that multirate transmission alternates among classes, depending on which QoS region, whether low or high, the users are adhering to, as well as on the total number of users exploiting the system and their distribution among those classes.

\section{APPENDIX}

Proof of Lemma 2: Consider that $\mathbf{R}^{*}=\left(R_{i}^{*}, R_{j}^{*}\right)^{\mathrm{T}}$ solves the optimization problem $\left(\Pi_{2}\right)$ for any two-class subsystem: class- $i$ and class- $j \forall i \neq j \in\{0,1, \ldots, S-1\}$. Satisfying the KT conditions, the solution of $\left(\Pi_{2}\right)$ is represented by 12 possible cases. For each one of them, all the variables are feasible and have unique solution in finite intervals of the QoS $\beta_{i}$ and $\beta_{j}$ for class $-i$ and class- $j$, respectively. Consequently, the solution is given as follows:

Case 1:

$$
\beta_{i} \in\left(\beta_{j}, \frac{1}{M_{0} R^{(\ell)}}\right)
$$

and

$\beta_{j} \in\left(0, \frac{1-2 M_{i} R^{(\ell)} \beta_{i}-\sqrt{\left(1-2 M_{i} R^{(\ell)} \beta_{i}\right)^{2}-\left(2 M_{j} R^{(u)} \beta_{i}\right)^{2}}}{2 M_{j} R^{(u)}}\right)$

if the second term has a positive real value; otherwise, we have the expression of $\beta_{j}$, shown at the bottom of the page. The optimal solution is given by

$$
\begin{aligned}
& R_{i}^{*}=R^{(\ell)} \\
& R_{j}^{*}=R^{(u)} .
\end{aligned}
$$

The Lagrange multipliers are

$$
\begin{aligned}
& \lambda_{1}^{*}=\operatorname{SNR}_{\mathrm{n}} M_{i}\left(2 M_{i} R^{(\ell)}+\left(\frac{\beta_{i}}{\beta_{j}}+\frac{\beta_{j}}{\beta_{i}}\right) M_{j} R^{(u)}-\frac{1}{\beta_{i}}\right) \\
& \lambda_{4}^{*}=\operatorname{SNR}_{\mathrm{n}} M_{j}\left(-2 M_{j} R^{(u)}-\left(\frac{\beta_{i}}{\beta_{j}}+\frac{\beta_{j}}{\beta_{i}}\right) M_{i} R^{(\ell)}+\frac{1}{\beta_{j}}\right) \\
& \lambda_{2}^{*}=\lambda_{3}^{*}=\lambda_{5}^{*}=0 .
\end{aligned}
$$

By checking the interval of $\beta_{i}$ and $\beta_{j}$, we can easily recognize that $\beta_{i}>\beta_{j}$. In addition, the optimal solution in (27) and (28) shows clearly that $R_{i}^{*}<R_{j}^{*}$, which satisfies Lemma 2.

Case 2:

$$
\beta_{i} \in\left(\beta_{j}, \frac{1}{M_{i} R^{(\ell)}}\right)
$$

and

$\beta_{j} \in\left(\begin{array}{l}\frac{-M_{j} R^{(u)} \beta_{i}+\sqrt{\left(M_{j} R^{(u)} \beta_{i}\right)^{2}+M_{i} R^{(\ell)}\left(\beta_{i}-M_{i} R^{(\ell)} \beta_{i}^{2}\right)}}{M_{i} R^{(\ell)}}, \\ \min \left(\beta_{i}, \frac{-M_{j} R^{(\ell)} \beta_{i}+\sqrt{\left(M_{j} R^{(\ell)} \beta_{i}\right)^{2}+M_{i} R^{(\ell)}\left(\beta_{i}-M_{i} R^{(\ell)} \beta_{i}^{2}\right)}}{M_{i} R^{(\ell)}}\right)\end{array}\right)$

$R_{i}^{*}=R^{(\ell)}$

$R_{j}^{*}=\frac{1}{2 M_{j} \beta_{j}}-\frac{M_{i}}{2 M_{j}}\left(\frac{\beta_{i}}{\beta_{j}}+\frac{\beta_{j}}{\beta_{i}}\right) R^{(\ell)}$

$\lambda_{1}^{*}=\operatorname{SNR}_{\mathrm{n}} M_{i} \frac{\left(\beta_{i}^{2}-\beta_{j}^{2}\right)}{2 \beta_{i}^{2} \beta_{j}^{2}}\left(\beta_{i}-M_{i} R^{(\ell)}\left(\beta_{i}^{2}-\beta_{j}^{2}\right)\right)$

$\lambda_{2}^{*}=\lambda_{3}^{*}=\lambda_{4}^{*}=\lambda_{5}^{*}=0$.

Note that $\beta_{j}<\beta_{i}, R_{i}^{*}=R^{(\ell)}$, and $R_{j}^{*} \neq\left\{R^{(\ell)}, R^{(u)}\right\}$. In addition, since $R_{i}^{*}$ and $R_{j}^{*}$ are feasible solutions and satisfying Lemma $1, R_{j}^{*}$ should satisfy $R^{(\ell)} \leq R_{j}^{*} \leq R^{(u)}$. This implies that $R_{j}^{*}>R_{i}^{*}$.

The same reasoning can be applied to the remaining ten cases that correspond to different ranges of $\beta_{i}$ and $\beta_{j}$. As a result, it is clear that for all possible solutions of a two-class subsystem when $\beta_{i}>\beta_{j}, R_{i}^{*} \leq R_{j}^{*}$ and vice versa, which proves the lemma for the two subclasses. To generalize this result to the $S$-class system, consider a third class, which we call class- $k$, with a QoS $\beta_{k}$. Applying the previous derivation to the twoclass subsystem class- $j$ and class- $k$, we can prove that when $\beta_{j}>\beta_{k}, R_{j}^{*} \leq R_{k}^{*}$, and vice versa. Therefore, if $\beta_{i}>\beta_{j}>$ $\beta_{k}$, then $R_{i} \leq R_{j} \leq R_{k}$ is always true. Following the same recurrence for $S$ classes, we can easily show that if $\beta_{0}>\beta_{1}>$ $\cdots>\beta_{S-1}$, then $R_{0} \leq R_{1} \leq \cdots \leq R_{S-1}$, which completes the proof.

$$
\beta_{j} \in\left(0, \frac{-M_{j} R^{(u)} \beta_{i}+\sqrt{\left(M_{j} R^{(u)} \beta_{i}\right)^{2}+M_{i} R^{(\ell)}\left(\beta_{i}-M_{i} R^{(\ell)} \beta_{i}^{2}\right)}}{M_{i} R^{(\ell)}}\right)
$$




\section{REFERENCES}

[1] J. A. Salehi and C. A. Brackett, "Code division multiple-user techniques in optical fiber networks-Part I," IEEE Trans. Commun., vol. 37, no. 8, pp. 824-833, Aug. 1989.

[2] H. M. H. Shalaby, "Complexities, error probabilities, and capacities of optical OOK-CDMA communication systems," IEEE Trans. Commun., vol. 50, no. 12, pp. 2009-2017, Dec. 2002.

[3] J. G. Zhang, "Novel optical fiber code-division multiple access networks supporting real-time multichannel variable-bit-rate (VBR) video distributions," IEEE Trans. Broadcast., vol. 43, no. 3, pp. 339-349, Sep. 1997.

[4] J. G. Zhang, "Design of a special family of optical CDMA address codes for fully asynchronous data communications," IEEE Trans. Commun., vol. 47, no. 7, pp. 967-973, Jul. 1999.

[5] L. C. Yun and D. G. Messerschmitt, "Variable quality of service in CDMA systems by statistical power control," in Proc. IEEE ICC, Seattle, WA, 1995, pp. 713-719.

[6] M. L. Honig and J. B. Kim, "Resource allocation for packet data transmission in DS-CDMA," in Proc. 33rd Allerton Conf. Commun., Control, Comput., Urbana, IL, 1995, pp. 925-934.

[7] S. Ramakrishna and J. M. Holtzman, "A scheme for throughput maximization in a dual class CDMA system," IEEE J. Sel. Areas Commun., vol. 16, no. 6, pp. 830-844, Aug. 1998.

[8] E. Inaty, H. M. H. Shalaby, P. Fortier, and L. A. Rusch, "Multirate optical fast frequency hopping CDMA system using power control," J. Lightw. Technol., vol. 20, no. 2, pp. 166-177, Feb. 2002.

[9] H. Yashima and T. Kobayashi, "Optical CDMA with time hopping and power control for multirate networks," J. Lightw. Technol., vol. 21, no. 3, pp. 695-702, Mar. 2003.

[10] T. Miyazawa and I. Sasase, "Multi-rate and multi-quality transmission scheme using adaptive overlapping pulse-position modulator and power controller in optical network," in Proc. IEEE ICON, Nov. 2004, vol. 1, pp. $127-131$.

[11] N. G. Tarhuni, M. S. Elmusrati, T. O. Korhonen, and E. Mutafungwa, "Multi-access-interference mitigation using power control in opticalCDMA star networks," in Proc. IEEE Int. Control Conf., May 2005, vol. 3, pp. 1593-1597.

[12] E. Inaty, H. M. H. Shalaby, and P. Fortier, "On the cutoff rate of a multiclass OFFH-CDMA system," IEEE Trans. Commun., vol. 53, no. 2, pp. 323-334, Feb. 2005.

[13] E. Inaty, H. M. H. Shalaby, and P. Fortier, "A new transmitter-receiver architecture for noncoherent multirate OFFH-CDMA system with fixed optimal detection threshold," J. Lightw. Technol., vol. 20, no. 11, pp. 1885-1894, Nov. 2002.

[14] L. D. Wronski, R. Hossain, and A. Albicki, "Extended hyperbolic congruencial frequency hop code: Generation and bounds for cross- and autoambiguity function," IEEE Trans. Commun., vol. 44, no. 3, pp. 301-305, Apr. 1996.

[15] E. K. P. Chong and S. H. Zak, An Introduction to Optimization. New York: Wiley-Interscience, 2001

[16] L. Cooper, Applied Nonlinear Programming for Engineers and Scientists. Englewood Cliffs, NJ: Aloray, 1974.

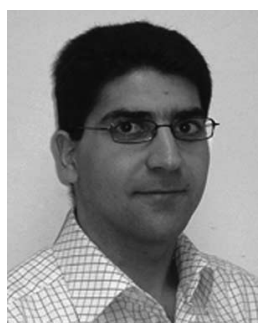

Robert Raad was born in Rahbé, Lebanon, on August 24, 1978. He received the B.S. and M.S. degrees in computer engineering from the University of Balamand, El-Koura, Lebanon. He is currently working toward the Ph.D. degree at Laval University, Quebec City, QC, Canada.

His research interests include optical code division multiple access and network control and resource management issues in optical communications networks.

Mr. Raad received an award from the professors of the faculty of engineering at the University of Balamand in 2003.

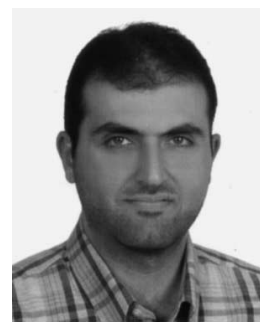

Elie Inaty (S'99-M'02) was born in El-Koura, Lebanon, on June 23, 1975. He received the B.S. and M.S. degrees in electrical engineering from the University of Balamand, El-Koura, in 1996 and 1998, respectively, and the $\mathrm{Ph} . \mathrm{D}$. degree from Université Laval, Quebec City, QC, Canada, in 2001.

$\mathrm{He}$ is currently an Assistant Professor at the University of Balamand and an Adjunct Professor at Université Laval. His research interests include code division multiple access and wavelength-divisionmultiplexing fiber optic communications, network control and resource management issues in optical communication networks, and radio multiple-access techniques.

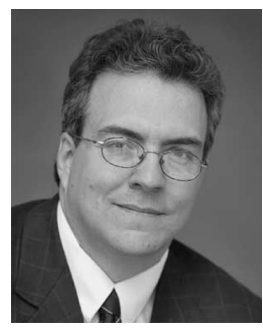

Paul Fortier (S'79-M'82-SM'00) received the B.Sc. and M.Sc. degrees in electrical engineering from Laval University, Quebec City, QC, Canada, in 1982 and 1984, respectively, and the M.S. degree in statistics and Ph.D. degree in electrical engineering from Stanford University, Stanford, CA, in 1987 and 1989, respectively.

Since 1989, he has been with the Department of Electrical and Computer Engineering, Laval University, where he is currently a Full Professor. From 1991 to 1997, he was Program Director for the B.Sc. degree in computer engineering, and from 1997 to 2003, he was Chairman of the Department of Electrical and Computer Engineering. Since 2003, he has been Associate Dean for development and research at the Faculty of Science and Engineering. He has done consulting work for several companies and government agencies in Canada. His research interests include digital signal processing for communications and the study of complexity and performance tradeoffs in hardware implementations, with applications in wireless and optical communications. He has been involved in the organization of national and international conferences and workshops in these fields.

Dr. Fortier is a Fellow of the Engineering Institute of Canada.

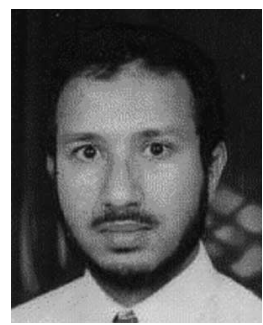

Hossam M. H. Shalaby (S'83-M'91-SM'99) was born in Giza, Egypt, in 1961. He received the B.S. and M.S. degrees from the University of Alexandria, Alexandria, Egypt, in 1983 and 1986, respectively, and the Ph.D. degree from the University of Maryland, College Park, in 1991, all in electrical engineering.

In 1991, he was with the Department of Electrical Engineering, University of Alexandria, as an Assistant Professor. He was promoted to an Associate Professor in 1996 and to a Professor in 2001 (current position). Since December 2000, he has been an Adjunct Professor with the Department of Electrical and Information Engineering, Faculty of Sciences and Engineering, Laval University, Quebec City, QC, Canada. From March to April 1996, he was a Visiting Professor with the Electrical Engineering Department, Beirut Arab University, Beirut, Lebanon. From September 1996 to January 1998, he was an Associate Professor with the Electrical and Computer Engineering Department, International Islamic University Malaysia, and from February to December 1998, he was with the School of Electrical and Electronic Engineering, Nanyang Technological University, Singapore, where he was a Senior Lecturer, and from January 1999 to February 2001, an Associate Professor. His research interests include optical communications, optical code division multiple access, spread-spectrum communications, and information theory. 